\title{
Research of the Communication Middleware of the Yacht Supervision Management System Based on DDS
}

\author{
Yan-Ru WANG ${ }^{1}$, Yi-Kun ZHANG ${ }^{2}$, Song-Yuan GU ${ }^{2}$,Wei-Feng XU ${ }^{3}$ \\ ${ }^{1}$ Economics School of Jilin University, Jilin University, 2699 Qianjin Street, Changchun, 130012, China \\ 2 College of Computer Science and Technology, Jilin University, 2699 Qianjin Street, Changchun, 130012, China \\ ${ }^{3}$ School of Control and Computer Engineering, North China Electric Power University, 619 Yonghua North Street, Baoding, 071003, China
}

\begin{abstract}
The communication middleware of the yacht supervision management system (YSM) which is based on the DDS is the communication management software of the yacht supervision center system and the ship monitoring system .In order to ensure the high efficiency and high reliability of communication middleware, the paper for the first time introduced DDS communication framework to the yacht supervision system in the process of software design and implementation, and designed and implemented more flexible and reliable communication management interface for the DDS communication framework. Through practical test, each performance index of DDS communication middleware software of the yacht supervision management system has reached the design requirements.
\end{abstract}

\section{Introduction}

Currently, marine resources have been vital bases for the further development of countries in the world. China is also at a crucial period of the development strategy of marine resources. The strategic objective of building maritime power which proposed at the report to Eighteenth CPC National Congress, offered a golden development opportunity and political guarantee to research, exploit and utilize the marine resources of China's marine related industries. China's marine related industries cover many trades including energy, scientific research, resources and tourism etc. The marine tourism plays an important role in marine related industry, which based on plentiful travel resources, various types and high-grade tourism products, broad and comfortable tourism activities, and unique content of tourism culture. Currently, since enormous advantages the yacht tourism takes in consumption levels and brand effect, it is gradually turning into a new jumpingoff point for marine tourism, and have been proven to be an important segment of China's marine strategy at the meanwhile[1].

The communication middleware of the yacht supervision management system (YSM) which based on the DDS is the communication management software of the yacht supervision center. In supervision center, this software complete functions including information sending, receiving and recording, replaying, transferring in different means of communication and trafficking statistics between supervision center and ships[2-4]. The software also offers timing services and the management function of various communication devices for yacht supervision center and the ship monitoring system. The software can make one communication device serves multiple business systems, and lay a foundation for the forming of the distributed internal system for supervision center or larger yacht.

The communication middleware of the yacht supervision management system (YSM) based on DDS that we research and implement will build on RTI-DDS communication middleware, to design and implement the communication interface which is fit for the application of this software, to implement the functions of transmittingreceiving information, switching different communication mode, forwarding information, recording and replaying information, trafficking statistics, managing devices, configuring software, selecting channels, issuing logs and displaying the transmitted-received information.

\section{DDS Theoretical Model}

\subsection{Publish/subscribe Model}

Publish/subscribe model was a messaging communication middleware proposed by OMG (Object Management Group) based on real-time communication of distributed system[5]. This is the only messaging communication mode so far which able to meet the time, space, and loosely coupled of 
data transmission at the meanwhile. OMG has been issued a latest standard based on publish/subscribe communication mode called Data Distribution Service (DDS). The specification carries out data distribution completely based on data-centric, meanwhile, define and implement QoS strategy, achieve a unified description of resources state, the level of resources expectation and network state. Thus enable the control to system, and satisfy the flexibility and timeliness of the real-time communication[6-7].

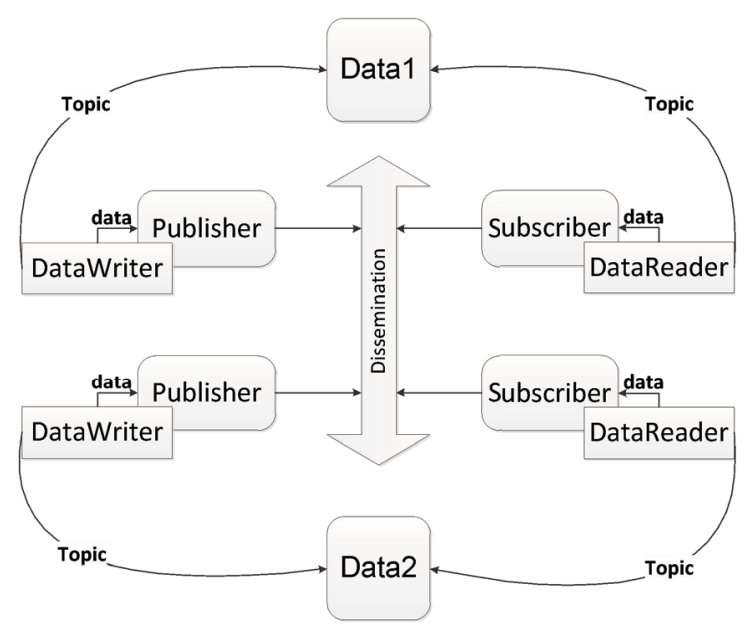

Figure 1 Basic Implementation Architecture of Dds Model

There are many data transmit system software achieved by publish/subscribe mechanism. Currently, perfect DDS products are RTI-DDS, ZHDDS, OpenDDS and Opensplice-DDS[8-10]. The paper implement the improvement of data transmit mechanism mainly based on RTI-DDS open source, and also introduce the important architecture and implementation mechanism of OpenDDS. OpenDDS has the advantage of single-node control and easy to configuration, which can significantly reduce the workload of environment configuration before use. Yet DCPSInfoRepo has to run on a separate node because of the internal implementation mechanism, so it is easy to have the problem of single point failure, and then get to performance bottleneck.

Data Distribution Service(DDS) model is a data communication standard with high real-time performance and high reliability, including two part, Data-Centric Publish-Subscribe(DCPS) layer and Data Local Reconstruction layer(DLRL) respectively. DCPS layer is the basic layer, constraint to the QoS policy strictly, and establish the application programming interface. DLRL layer built on the top of DCPS layer, and it is optional, allows local object to share transmission data like to share local data.

The basic implementation architecture of DDS data distribution service model is shown in Figure 1, which mainly involves the following members: Publisher which is responsible for data publish, Subscriber which is responsible for data subscriptions, DataWriter which is responsible for data writing and DataReader which is responsible for data reading.

\subsection{RTI DDS}

Network data distribution services NDDS is a data distribution middleware product of RTI, compliant with the OMG's DDS standard completely. The NDDS middleware of RTI has been integrated with DDS API, all of data supply and collection program can still transfer data through a fixed API interface even after the system's communication devices upgraded or extended. For instance, a GPS sensor is publishing location information through DDS, RTI-DDS can offer an estimation of location information as an alternative when sensor paused, and information subscriber could not feel any change about it. The characteristic about transparent of data transmission is obtained by the standard API of decoupled data transmission and data application, which offered by RTIDDS. Application can focus on solving the problem of the application layer with regardless of the data pass pattern specifically. RTI-DDS is a good choice for developers who need to implement data-centric published order communication network, particularly when these developers need the network to have the properties of high efficiency, ease of implementation, platform-independent and language-independent.

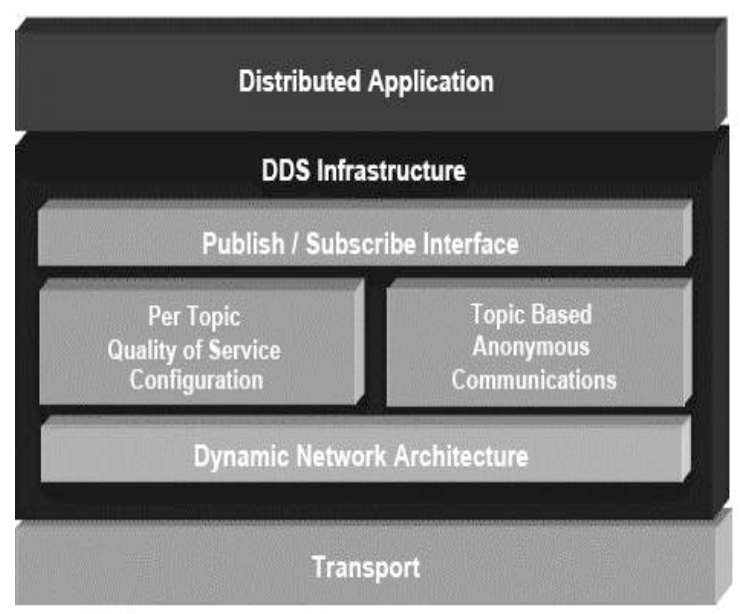

Figure 2 Transmission Architecture of Rti-Dds

\section{Design of Communication Middleware}

The communication middleware of the yacht supervision management system (YSM) which based on the DDS is the communication management software of the yacht supervision center. In supervision center, this software complete functions including information sending, receiving and recording, replaying, transferring in different means of communication and trafficking statistics between supervision center and ships. The software also offers timing services and the management function of various 
communication devices for yacht supervision center and the ship monitoring system.

\subsection{System Workflow}

System workflow is designed based on system requirements analysis. The main workflow of communication middleware is showed by Figure 3. As the figure shows, the system needs functional configuration before the software starts, and produces software functional configuration files after the software being functional configured. Software functional configuration is mainly a selection to system function. It helps improve the actual runtime efficiency of the system when we proceed functional configuration to software. We need configure the communication parameters and information forward relationship of communication equipment and produce configuration files of parameters and information forward relationship before the software starts. After starting the software, the system will start the related communication and location devices according to the configuration files, initialize communication interface, and establish information forward relation. As for information recording, traffic statistics, information playbacking, and communication channel, you can use the default settings or set up separately.

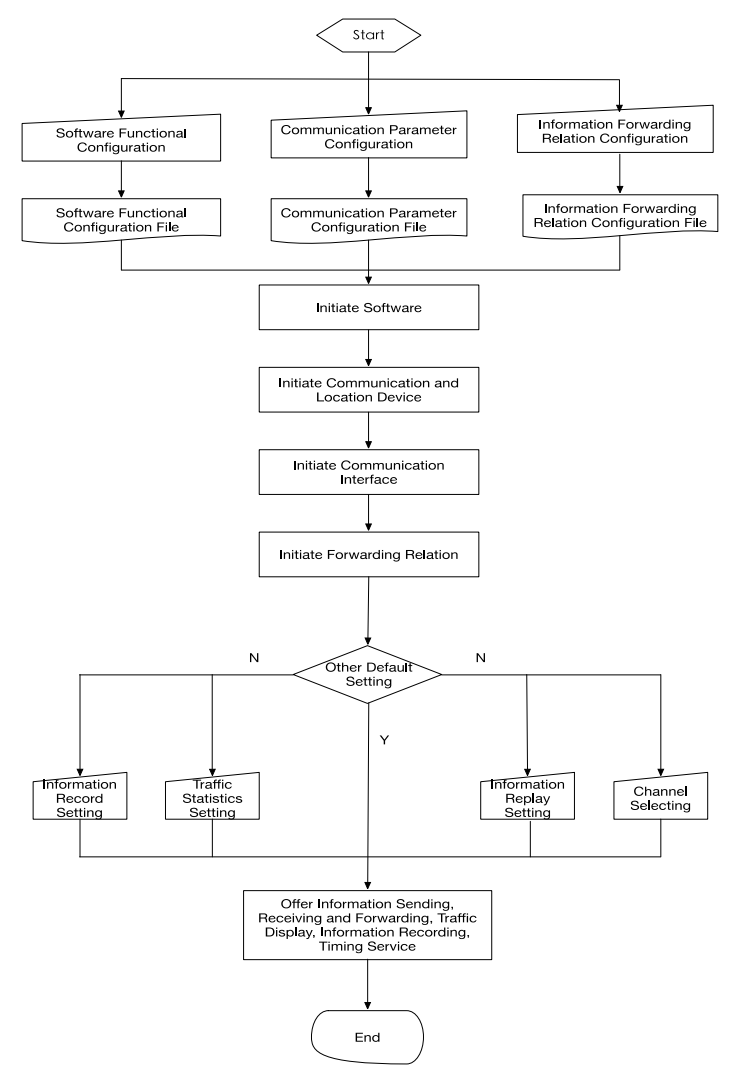

Figure 3 Workflow Diagram of Communication Middleware

\subsection{Design of System Architecture}

The basic architecture of the communication middleware of the yacht supervision management system based on DDS reflect the fundamental common properties of the software and offer an extension point which reflect changeable and updateable of the software. The common properties of the software can be showed by the fundamental architecture level and public components in Figure 4, the extension point mainly refers to software's update and the flexible extend of component. The basic architecture of this software is based on components. The lowest layer of architecture is composed of DDS communication component, Ethernet communication component, serial communication component and communication management component. Concrete business function layer is composed of information record, traffic statistics and function component of wireless communication devices. The top layer is an interface which offers all kinds of communication services to external user. In the software design process, we design component as a module, we will give a specific design in later.

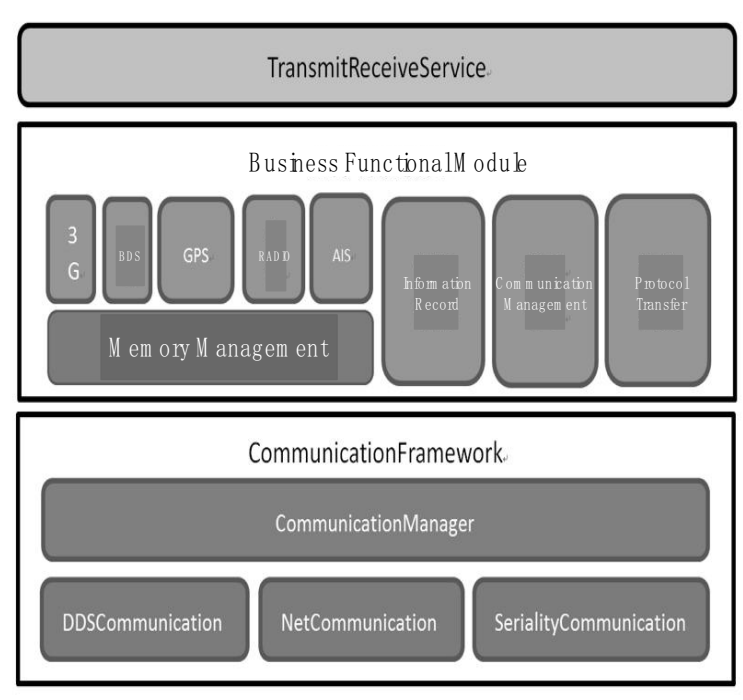

Figure 4 System Architecture of Communication Middleware

\subsection{Design of System Transfer Link}

Since the use of DDS in the communication middleware of the yacht supervision management system based on DDS, we must analyze the flow in the transfer software of data. We define the link which information enter the yacht supervision center system and the ship monitoring system through wireless communication devices as Information Input Link, the contrary information link is Output Link.

Information input link is showed in Figure 6. In this link, data is received by the receiving equipment of BDS (BeiDou Navigation Satellite System), GPS and radio, and pass into the system through the serial port, confirm the receiving information's internal system (the yacht supervision center system which deployed on different seats 
and the ship monitoring system) through data process and receive-send mapping, and then transform the information into DDS information format and send it to the internal network. The data received by external network and $3 \mathrm{G}$ receiving equipment will be passed into system through Ethernet, after which the process is the same as above. Figure 6 shows the information output link, the information flow is at opposite direction with the process above, the detailed process has been shown clearly in Figure.

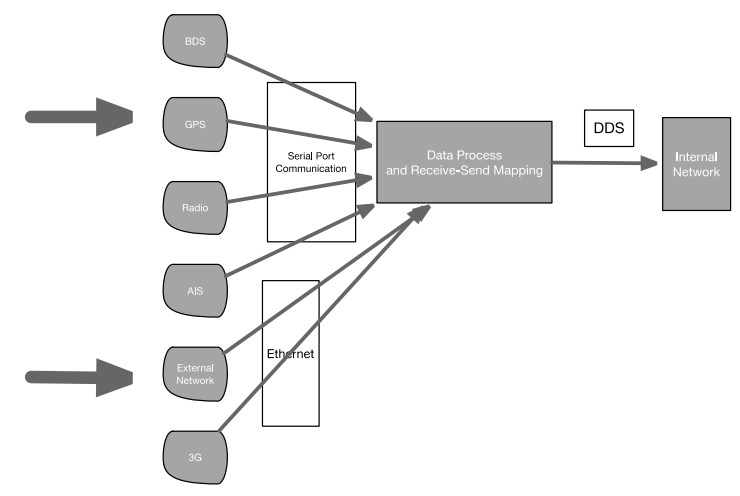

Figure 5 Information Input Link

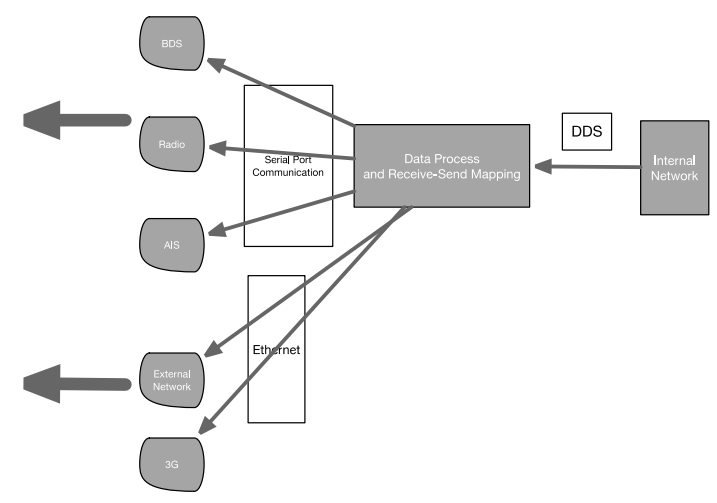

Figure 6 Information Output Link

The design of communication link above can make a communication device serves several business systems, laid the foundation to develop a distribution's internal system of supervision center or a larger yacht.

Since the communication middleware of the yacht supervision management system based on DDS also offered management function to all types of communication devices for the yacht supervision center system and the ship monitoring system, so we need to analyse and design the device control link. The ship monitoring system can control and manage the communication devices of this ship according to the requirements, also, supervision center can get some basic control to the communication device when ship system can't control the devices or require supervision center to control the communication device. The control information link is showed in Figure 7.

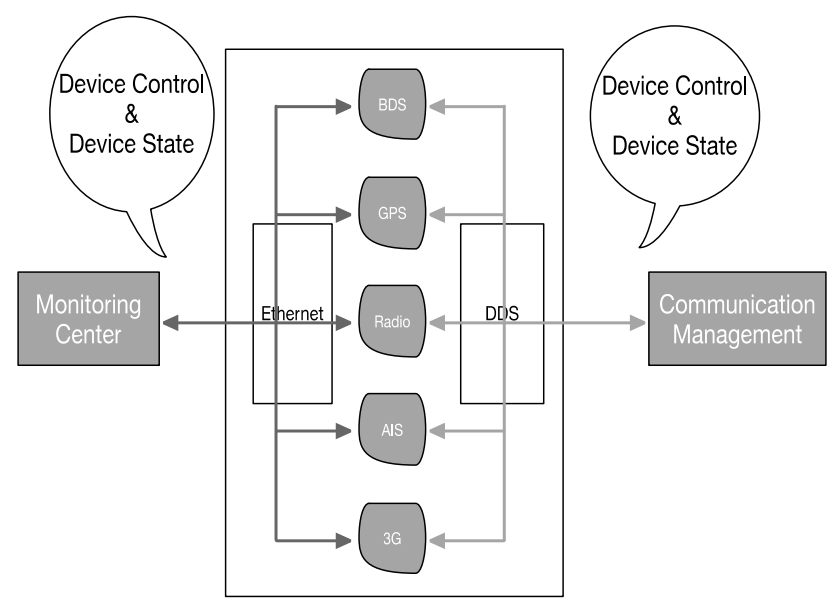

Figure 7 Device Control Link

\section{Implementation and Test}

\subsection{Implementation of Communication Middleware}

The communication middleware of the yacht supervision management system based on DDS is an underlying communication management software, so it has higher demand for running efficiency and stability. At present, the language for developing this software are $\mathrm{C}++, \mathrm{C} \#$, JAVA and so on, the development conditions are also various.

Currently, the communication middleware of the yacht supervision management system based on DDS which implemented in this paper has been test running at a sea experimental base. During the test run, the scene was set to have a supervision management center and five yachts, there were four monitoring seats set around the monitoring center and configured related supervision management system, each yacht equipped a yacht supervision management software, monitoring center configured with a communication middleware, and each yacht configured with a communication middleware respectively.

What showed in Figure 8 is the practical running state of yacht supervision management center when it monitoring the four yachts. The upper part of this software is traffic statistics and display interface, the yellow curve shows the traffic of input information, the orange one shows the traffic of output information. At the lower right corner of the software interface shows the detail information, including information type, receiving and sending time and so on. The lower part of the software shows the detail information coming from different wireless communication device. 


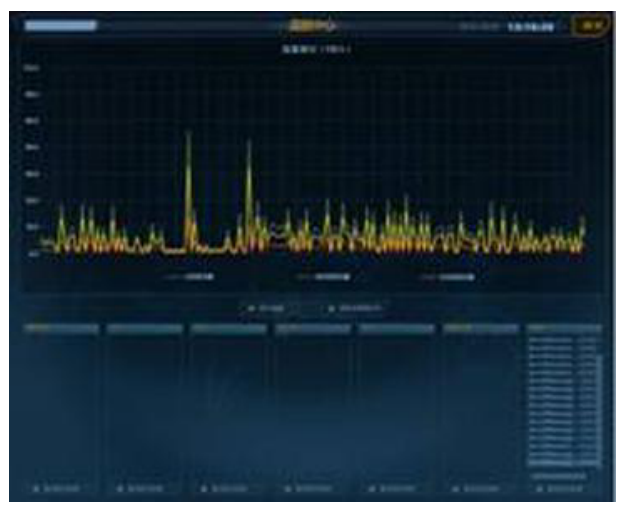

Figure 8 Running Diagram of Communication Middleware

\subsection{Test of Communication Middleware}

During the test process, we did unit test on transfer software first. During the test, the key functions were tested. Ultimately, $81 \%$ of the test cases passed test, $19 \%$ of the test cases failed. After a regression test, all the cases that didn't pass before had passed.

Then we took configuration item test on transfer software. The test types of configuration item include document review, functional test, static analysis, performance test, interface test, boundary test, intensity test, security test, recovery test and installation test.

After unit test and configuration item test, the software implemented in this paper achieve the following technical criterion: the throughput of the communication middleware of the monitoring center: $\geqslant 2 \mathrm{Gbps}$; the simultaneously sessions of the communication middleware of the monitoring center: $\geqslant 1000$; the throughput of the ship communication middleware: $\geqslant 100 \mathrm{Mbps}$; the simultaneously sessions of the ship communication middleware: $\geqslant 100$; the maximum connection number of roll call mechanism of each ratio modem: 50; the new establish connection number of naming mechanism of each ratio modem per second: $\geqslant 10$; time of software initiate: $<=$ $2 \mathrm{~s}$; time of each sub-interface switching at normal running state: $<=2 \mathrm{~s}$; time from receive some data correctly to forward the data through DDS successfully at normal running state: $<=1 \mathrm{~ms}$; time from receive some data correctly to forward the data through UDP successfully at normal running state: $<=1 \mathrm{~ms}$; time from receive some data correctly to forward the data through a wireless communication device successfully at normal running state: $<=60 \mathrm{~ms}$; response time of primary channel switching: $<=200 \mathrm{~ms}$; time interval of device checking:1s; time from equipment error occurred to error confirmed: $<=1500 \mathrm{~ms}$; time from equipment error confirmed to initiate redundant equipment: $<=200 \mathrm{~ms}$; roll call time slice when ratio modem as the communication equipment: $<=200 \mathrm{~ms}$; roll call cycle when ratio modem as the communication equipment: $<=10 \mathrm{~s}$.

The technical criterion that this software achieved satisfy the software design requirements at the test process above, offering basis for software quality evaluation, and also offering technical criterion for software practical application.

\section{Summary and Outlook}

The communication equipment that yacht supervision management system(YSM)used is a multimode communication model with high reliability and efficiency, which consist of $3 \mathrm{G}, 4 \mathrm{G}$, satellite, radio, ship automatic identification system(AIS). On the basis of in-depth analysis to multiple products of DDS, in order to guarantee the stability, security and efficiency of the software installed in the yacht supervision management system, we choose RTI-DDS as the communication middleware to implement the software in this paper, and design and implement a more flexible and more reliable communication management interface for RTI-DDS communication architecture. This paper achieves the preliminary design, detailed design, system implementation, system test of the communication middleware of the yacht supervision management system based on DDS and introduce the actual running situation. According to the test running situation, the communication middleware of the yacht supervision management system based on DDS which this paper design and implemented achieves the design goal and satisfies requirements.

Yacht supervision management is a complicated and could to be improved and updated information system according to market requirement, so the communication middleware of the yacht supervision management system based on DDS which this paper implemented need to be improved with respect of the update and upgrade of the YSM system. Currently, the software achieves the designed requirement, but adopt $3 \mathrm{G}$ communication forward interface while communication with $4 \mathrm{G}$, and did not test the $4 \mathrm{G}$ communication particularly. In summary, we will continue to update and upgrade the communication middleware, and perfect the $4 \mathrm{G}$ communication forward interface in our future work.

\section{References}

Zhang Cong. Research on the Development Strategy of Marine Eco-tourism Resources. Ocean University of China, 2009.

Ma Yuting. Construction of Chinese marine strategy in the new era. ZheJiang University, 2013.

Tim Harrison, Irfan Pyarali. An Object Behavioral Pattern for Demultiplexing and Dispatching Handlers for Asynchronous Events[C]. 4th Pattern Languages of Programming conference in Allerton Park, Illinois, September 2-5, 1997.

Kyoungho An, Aniruddha Gokhale. A Cloud-enabled Coordination Service for Internet-scale OMG DDS applications[C]. Proceedings of the 8th ACM International 
Conference on Distributed Event-Based Systems, New York, 2014.

1. P. Hunt, M. Konar, F. P. Junqueira, and B. Reed. Zookeeper: wait-free coordination for internet-scale systems [C]. In Proceedings of the 2010 USENIX conference on USENIX annual technical conference, volume 8, 2010, 11-11.

2. M. Li, F. Ye, M. Kim, H. Chen, and H. Lei. A scalable and elastic publish/subscribe service [C]. In Parallel \&Distributed Processing Symposium (IPDPS), 2011IEEE International, 2011, 1254-1265.

3. Mei Hong, Shen Jun-Rong, Progress of Research on Software Architecture. Institute of Software.

4. National institute of Standards and Technology. Recommended Security Controls for Federal Information Systems and Organizations[EB/OL]. https://www.researchgate.net/publication/238776571_ Recommended_Security_Controls_for_Federal_Inform ation_Systems_and_Organizations, 2009-09-14.

5. Real-Time Innovations. RTI Connext: Comprehensive SummaryofQoS Policies[EB/OL]. http://community.rti.com/documentation, 2013.

6. Real-Time Innovations. RTI Connext DDS: Core Libraries and Utilities User's Manual[EB/OL]. http://community.rti.com/documentation, 2013. 